Table DR1. Biostratigraphic and age assignments of successful paleomagnetic sites/sections from the Crotone basin.

\begin{tabular}{|c|c|c|c|c|c|c|}
\hline Site / Section & Formation & Calcareous nannofossils (MNN) & Planctonic foraminifera (MPL) & Chrons & Stage & Age (Ma) \\
\hline 1- ALLI (site) & Not defined & MNN12-15 & MPL2 & C3n & Zanclean & $5.23-4.18$ \\
\hline 2- FEGATO (site) & Not defined & MNN12-15 & MPL2 & C3n.4n-C3n.2n & Zanclean & $5.1-4.6$ \\
\hline 3- SIMERI (site) & Not defined & MNN1315 & Upper MPL3-LowerMPL 4 & $\mathrm{C} 2 \mathrm{Ar}$ & Zanclean & $4.2-3.6$ \\
\hline 4- M. BASILICATA (section 27 m-thick) & Cavalieri Marls & Not determined & MPL2 & C3n.4n-C3n.2r & Zanclean & $\begin{array}{l}\text { Base at } \sim 5.2 \\
\text { Top at } \sim 4.7\end{array}$ \\
\hline 5-COLLE MONICA (section 20 m-thick) & Cavalieri Marls & Not determined & MPL3 & C3n.1r or C2Ar & Zanclean & $\begin{array}{l}\text { Base at } \sim 4.5 \\
\text { Top at } \sim 4.0\end{array}$ \\
\hline 6- BOTRICELLO SW (site) & Cutro Clays & MNN16-18 & MPL5a & $\mathrm{C} 2 \mathrm{r}$ & Gelasian & $2.58-2.3$ \\
\hline 7- BOTRICELLO SUP. (site) & Cutro Clays & MNN18-19a & MPL6 & C2r.1r- Cn2 & Gelasian & $2.1-1.7$ \\
\hline 8- VACCARIZZO (section 57 m-thick) & Cavalieri Marls & Not determined & $\begin{array}{l}\text { From upper MPL1 to uppermost } \\
\text { MPL2 }\end{array}$ & C3n.4n-C3n.2r & Zanclean & $\begin{array}{l}\text { Base at } \sim 5.23 \\
\text { Top at } \sim 4.62\end{array}$ \\
\hline 9- CASE FORCA (section 33 m-thick) & Cavalieri Marls & Not determined & $\begin{array}{c}\text { From upper MPL2 to upper } \\
\text { MPL3 }\end{array}$ & C3n.2n-C3n.1r & Zanclean & $\begin{array}{l}\text { Base at } \sim 4.6 \\
\text { Top at } \sim 4.4\end{array}$ \\
\hline 10- CASE FORCA EST (section 15 m-thick) & Cavalieri Marls & Not determined & Upper MPL3 & C3n.1r & Zanclean & $4.3 \pm 0.1$ \\
\hline 11- MARCUCCIO (section 35 m-thick) & Cutro Clays & From mid MNN19e to lower MNN19f & Not determined & C1r.1n-C1r.1r & Advanced Calabrian & $\begin{array}{l}\text { Base at } \sim 1.1 \\
\text { Top at } \sim 0.95\end{array}$ \\
\hline 12- GROSSO (site) & Cutro Clays & MNN18 & Not determined & $\mathrm{C} 2 \mathrm{r}$ & Gelasian & $2.5-1.95$ \\
\hline 13- LA CELSA NE (section 52 m-thick) & Cutro Clays & From top MNN19d to lower MNN19e & Not determined & C1r.2r & Advanced Calabrian & $\begin{aligned} & \text { Base at } \sim 1.24 \sim 1 . \\
& \text { Top at } \sim 1.1\end{aligned}$ \\
\hline 14- TUFILICA (section 28 m-thick) & Cutro Clays & MNN19c & Not determined & C1r.3r & Lower Calabrian & $\begin{array}{l}\text { Base at } \sim 1.66 \\
\text { Top at } \sim 1.46\end{array}$ \\
\hline 15- TACINA W (section 52 m-thick) & Cutro Clays & From top MNN19a to basal MNN19c & Not determined & C1r.3r & Lower Calabrian & $\begin{array}{rl}\text { Base at } ~ & 1.73 \\
\text { Top at } \sim 1.6\end{array}$ \\
\hline 16- TERA (site) & Cutro Clays & MNN19c & Not determined & C1r.3r & Lower Calabrian & $1.67-1.62$ \\
\hline 17- R. BERNARDA (site) & Cutro Clays & MNN19b & Not determined & $\mathrm{C} 1 \mathrm{r}$ & Lower Calabrian & $\sim 1.73-1.6$ \\
\hline 18- RAPANA' (site) & Cutro Clays & MNN19d & Not determined & $\mathrm{C} 1 \mathrm{r} .2 \mathrm{r}$ & Middle Calabrian & $1.46-1.23$ \\
\hline 19- GALA (site) & Cutro Clays & MNN18 & Not determined & $\mathrm{C} 2 \mathrm{r}$ & Gelasian & $\sim 2.5-2$ \\
\hline 20- CAVALIERI (site) & Cavalieri Marls & Not determined & Not determined & C3n-C2Ar & Zanclean & $5.3-3.6$ \\
\hline 21- ZINGA (site) & Cavalieri Marls & Not determined & Not determined & $\mathrm{C} 3 \mathrm{n}-\mathrm{C} 2 \mathrm{Ar}$ & Zanclean & $5.3-3.6$ \\
\hline 22- SETTE (site) & Cutro Clays & MNN19b & Not determined & ? & Lower Calabrian & $\sim 1.7-1.6$ \\
\hline 23- BUCA (site) & Cutro Clays & MNN19b & Not determined & C1r.3r & Lower Calabrian & $\sim 1.7-1.6$ \\
\hline 24- BONA (site) & Cutro Clays & Not determined & Not determined & $\mathrm{C} 2 \mathrm{Ar} ?$ & Zanclean? & $4.2-3.6$ \\
\hline 25- PIANO (site) & Cutro Clays & MNN19d & Not determined & C1r.2r & Middle Calabrian & $1.46-1.23$ \\
\hline 26- SERRE (site) & Cutro Clays & MNN19d & Not determined & C1r.2r & Middle Calabrian & $1.46-1.23$ \\
\hline 27- TRIVIO (site) & Cutro Clays & From upper MNN17 to top MNN18 & Not determined & $\mathrm{C} 2 \mathrm{r}$ & Gelasian & $2.58-1.95$ \\
\hline 28- PAGA (site) & Cutro Clays & MNN17 & Not determined & C2An.1n & Piacenzian & $2.75-2.58$ \\
\hline 29- STRONGOLI (basal $10 \mathrm{~m}$ of a $123 \mathrm{~m}$-thick section) & Cutro Clays & MNN17 & MPL5a & C2An.1n & Top Piacenzian & $\sim 2.6$ \\
\hline
\end{tabular}

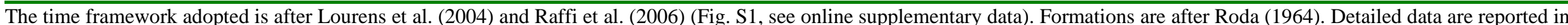
this paper for magnetic polarity and in online supplementary data (Database 1) for calcareous plankton biostratigraphic data. 


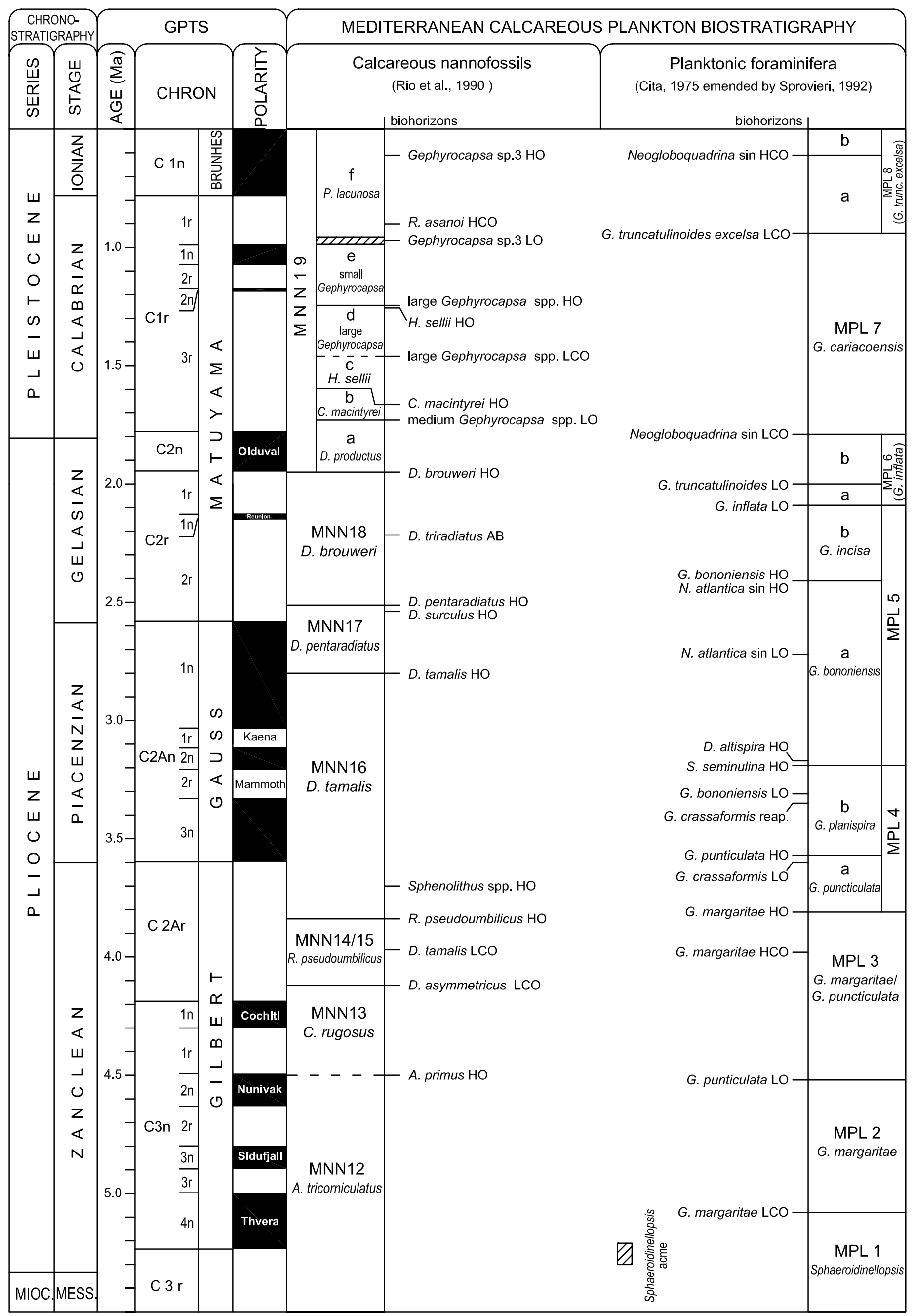




\section{Figure Caption}

3 Figure DR1. Adopted time framework after Lourens et al. (2004) and Raffi et al. (2006; for the 4 calcareous nannofossil biochronology).

$6 \quad$ Kr-Database.xls 